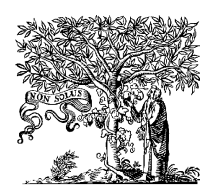

ELSEVIER

\title{
Chiral single crystal surface chemistry
}

\author{
Andrew J. Gellman*, Joshua D. Horvath, Mark T. Buelow \\ Department of Chemical Engineering, Carnegie Mellon University, Pittsburgh, PA 15213, USA
}

Received 4 April 2000

\begin{abstract}
Several experiments have been performed to probe the enantiospecific properties of chiral single crystal surfaces. The surfaces chosen have been the (643) planes of $\mathrm{Ag}$ and $\mathrm{Cu}$, both face centered cubic structures. The chirality of these surfaces arises from the handedness of their kinked step structures. These structures are such that the (643) and the $(\overline{643})$ surfaces are related by mirror symmetry but are non-superimposable. We denote them as $(643)^{R}$ and $(643)^{S}$. As a consequence of this handedness it is expected that the interactions of these surfaces with the left- and right-handed enantiomers of a chiral molecule should be different. In other words the chemistry of chiral molecules on these surfaces should be enantiospecific. We have observed that the desorption energies of $R$-3-methyl-cyclohexanone differ by $0.22 \pm 0.05 \mathrm{kcal} / \mathrm{mole}$ on the $\mathrm{Cu}(643)^{R}$ and the $\mathrm{Cu}(643)^{S}$ surfaces. Similarly, on the $\mathrm{Ag}(643)^{R}$ surface we have observed that the orientations of $R$ - and $S$-2-butanoxy groups differ. This enantiospecific orientation is revealed by the intensities of the absorption bands in an infrared absorption spectra of these species on the $\operatorname{Ag}(643)^{R}$ surface. These two results expand the small but growing set of observations of the enantiospecific properties of chiral single crystal surfaces. (C) 2001 Elsevier Science B.V. All rights reserved.
\end{abstract}

Keywords: Chiral surface; Enantioselective catalysis; High miller index

\section{Introduction}

Chirality is one of the most subtle of molecular properties and yet is of extreme importance in nature and in life. Most biological molecules are chiral in the sense that they can, in principle, exist as two identical structures that are mirror images of one another but non-superimposable. These two forms are referred to as enantiomers and are related to one another in the same sense as the left hand is related to the right hand. Although the molecules can exist in two forms, nature has evolved such that only single enantiomeric forms of chiral molecules exist in living organisms. This is of enormous consequence in the field of pharmaceu-

\footnotetext{
${ }^{*}$ Corresponding author. Tel.: +1-412-268-3848; fax: +1-412-268-7139.

E-mail address: ag4b@andrew.cmu.edu (A.J. Gellman).
}

ticals because it means that the two enantiomers of a chiral pharmaceutical, although they may have the same nominal structure do not interact with living organisms in the same manner. While one enantiomer of the drug may have therapeutic properties the other can be toxic. As a result synthetic methods that can yield chiral products enantioselectively are in great demand $[1,2]$.

Heterogeneous catalytic synthesis of fine chemicals and complex chiral molecules is in its infancy. The enantioselective synthesis of such molecules requires environments that are themselves chiral and of a single handedness. This has generated a great deal of interest in the preparation of chiral materials and surfaces for use as heterogeneous catalysts. The most widely pursued approach to the preparation of enantioselective heterogeneous catalysts has been the use of chiral organic templates which bind to the surface 
of a catalyst and create a chiral environment in which catalytic reactions can occur. The most commonly studied chiral heterogeneous catalyst makes use of cinchonidine as the chiral template adsorbed onto the surfaces of a supported Pt catalyst. When such catalysts are used for the hydrogenation of molecules such as ethylpyruvate they can achieve enantiomeric excesses as high as ee $=94 \% .^{1}$

The focus of this study is another entirely different route to the formation of chiral surfaces. Starting with single crystalline materials one can create high Miller index surfaces with structures consisting of kinked steps separated by flat low Miller index terraces. These structures have no mirror planes and thus, cannot be superimposed upon their mirror images [3]. Hence, they are chiral. Such chiral surfaces ought to have enantiospecific properties in the sense that their interactions with the two enantiomers of a chiral molecule must different. They ought to exhibit enantioselective adsorption, enantioselective reaction kinetics, or enantioselectivity in the synthesis of chiral molecules from prochiral reactants. Although single crystalline materials can only ever be produced in modest quantities, in some respects they should have advantages over chiral surfaces based on templating with organic species. Chiral single crystalline surfaces based on metals or inorganic compounds will undoubtedly be far more chemically robust and will be stable to much higher temperatures than organically templated chiral surfaces. Although the surface chemistry of high Miller index planes remain largely unexplored there is now a body of data that is revealing their enantiospecific properties.

The original work articulating the fact that high Miller index surfaces of metal single crystals are chiral attempted to use two probe reactions to reveal enantiospecificity [3]. The surfaces used in that work were the $\operatorname{Ag}(643)$ and $(\overline{643})$ planes which can be denoted $\operatorname{Ag}(643)^{R}$ or $\operatorname{Ag}(643)^{S}$ depending upon the orientations of their steps and kinks. Alternately, and more generally, the handedness can be defined on the basis of the orientations of the low Miller index planes which project through the surface as microfacets to form the step edges [4]. In the original work the handedness of the two surfaces was revealed quite clearly by the handedness of their low energy electron

\footnotetext{
${ }^{1}$ References to the use of cinchonidine and to the ee achieved.
}

diffraction (LEED) patterns. Demonstration of the enantiospecificity was attempted by measuring the desorption kinetics of $R$ - and $S$-2-butanol on each of the surfaces. A second reaction that was studied was the decomposition of $R$ - and $S$-2-butanoxy groups by $\beta$-hydride elimination. Unfortunately, in both cases the reaction kinetics did not reveal observable enantiospecificity. This placed an upper limit of roughly $0.1 \mathrm{kcal} / \mathrm{mole}$ on the differences in the desorption energies ( $\left.\Delta \Delta E_{\mathrm{des}}\right)$ of the $R$ - and $S$-2-butanols or the differences in the activation energies $\left(\Delta \Delta E_{\text {act }}\right)$ for $\beta$-hydride elimination in the $R$ - and $S$-2-butanoxy groups.

The first demonstration of the enantiospecific properties of chiral single crystal surfaces was via molecular simulation of the adsorption of chiral hydrocarbons on chiral Pt surfaces [5]. Those simulations used a potential developed to describe the scattering of hydrocarbons from $\mathrm{Pt}$ surfaces as the basis for a Monte Carlo simulation of the adsorption energies of the chiral molecules. The molecules chosen for simulation included species such as trans1,2-dimethyl-cycloalkanes and limonene. The results revealed that the enantiospecificity of the adsorption energies could vary from 0 to $2 \mathrm{kcal} /$ mole $[5,6]$.

The first experimental observation of the enantiospecificity of chiral single crystal surfaces came from the field of electrochemistry [4,7]. Pt electrodes can be prepared in aqueous solution in clean single crystalline form. A number of such electrode surfaces including the flat Pt(111), a stepped (but not kinked) $\mathrm{Pt}(211)$ surface, and most importantly the chiral $\mathrm{Pt}(643)$ and $\mathrm{Pt}(531)$ surfaces were used. The test reaction was the electrooxidation of $\mathrm{D}$ - and L-glucose in aqueous solution. As expected the achiral Pt(111) and $\mathrm{Pt}(211)$ surfaces did not reveal any enantioselectivity in the glucose oxidation kinetics. In contrast the chiral $\operatorname{Pt}(643)$ and $\operatorname{Pt}(531)$ surfaces yielded the very satisfying observation that the electrooxidation kinetics of glucose are enantiospecific. The rate of oxidation differed by as much as a factor of 2-3 depending upon the handedness of the reactant, or the handedness of the surface.

This paper adds two additional observations of enantiospecific surface chemistry to the growing list of examples. The first is the result of a study of the desorption kinetics of $R$-3-methyl-cyclohexanone on the $\mathrm{Cu}(643)^{R}$ and $\mathrm{Cu}(643)^{S}$ surfaces. The second 
example is the preliminary result of a study of the orientations of $R$ - and $S$-2-butanoxy on the $\operatorname{Ag}(643)^{R}$ surface using Fourier transform-infrared reflection absorption spectroscopy (FT-IRRAS).

\section{Experimental}

The experiments were performed in two different UHV surface analysis chambers. Both chamber were pumped into the $10^{-10}$ Torr range using cryopumps and titanium sublimation pumps. Each was equipped with $\mathrm{Ar}^{+}$ion sputter guns for cleaning the sample surfaces, leak valves for introducing organic vapors for adsorption onto the surfaces, and LEED optics for determining the surface orientation and order. The samples were mounted on UHV manipulators that allowed them to be moved for positioning in front of the various devices in the chamber. In addition the samples could be heated resistively and cooled with liquid nitrogen over a temperature range of 100-1000 K using a computer to maintain temperature control. The sample temperatures were measured using chromel-alumel thermocouples spotwelded to their edges.

The chamber used for the thermally programmed desorption experiments was equipped with a Dycor MA200M quadruple mass spectrometer for the desorption measurements and a CLAM II hemispherical analyzer used to obtain Auger electron spectra of the surface. All spectra were obtained using $\mathrm{Cu}(643)^{R}$ and $\mathrm{Cu}(643)^{S}$ surfaces that had been sputter cleaned and then annealed. The surfaces were exposed to the 3-methyl-cyclohexanone from the background with the sample temperature at $100 \mathrm{~K}$. The samples were then positioned in front of the mass spectrometer and heated at a constant rate of $1 \mathrm{~K} / \mathrm{s}$ while monitoring the signal in the mass spectrometer at $m / q=39$.

The chamber used for the FT-IRRAS experiments was fitted with a Mattson RS-1 FTIR spectrometer and has been described in some detail elsewhere [8]. The preparation of the sample surfaces involved sputter cleaning followed by exposure to $100 \mathrm{~L}$ of $\mathrm{O}_{2}$ at $300 \mathrm{~K}$ using a line-of-sight doser. The surface was then exposed to 0.21 of $R$ - or $S$-2-butanol at $210 \mathrm{~K}$. At that temperature the 2-butanols are deprotonated by the surface oxygen which desorbs as water leaving the 2-butanoxy groups on the surface [3]. The FT-IRRAS spectra were obtained using 5000 scans with a resolu- tion of $4 \mathrm{~cm}^{-1}$. The sample surface was then heated to $400 \mathrm{~K}$ to remove the 2-butanoxy groups before taking the background spectrum of the clean surface.

The $\mathrm{Cu}(643)$ surface was obtained commercially and used as received. Note that since these samples are disks cut from single crystal boules, if one side exposes a (643) surface, then the other side must be the (543) surface. In other words the two sides of such single crystal samples expose surfaces of opposite handedness. The $\operatorname{Ag}(643)$ samples was cut from a single crystal boule as described earlier and also exposed surfaces of opposite handedness on either side [3].

The chemicals were purchased commercially. Before used they were purified by cycles of freezing, pumping and thawing to remove any high vapor pressure contaminants.

\section{Results}

The observation of subtle enantiospecific effects in the chemistry of chiral surfaces requires some attention to experimental design. One advantage of the use of the single crystal disks described above is that experiments on the two surface enantiomers can be performed on the same sample by simply using the front and the back faces, one after the other. This eliminates the need for the use of two separate samples and remounting of the sample when one wishes to switch surfaces. The sample temperature is measured by one thermocouple. This makes it feasible to perform experiments in which one is measuring temperatures reproducibly with an accuracy of $<1 \mathrm{~K}$. It should be noted that even in these ideal circumstances, care must be taken. We found that even in the desorption of achiral molecules from the two sides of the sample, differences of $1-2 \mathrm{~K}$ could be observed in the peak desorption temperatures if the surfaces were not equally clean or if the heating rates were too high.

It is necessary to have well established control experiments in order to ascertain the level to which one can be confident of observed differences in the surface chemistry of chiral species on chiral surfaces. If both enantiomers of a chiral species are available (and of the same purity) then it is possible to perform control experiments in which both the $R$ - and the $S$-enantiomers of the adsorbate are studied on the $R$ - and the $S$-surfaces. In principle, the results from 
the $R / R$ and the $S / S$ adsorbate/substrate combination should be identical and the results from the $R / S$ and the $S / R$ combination should be identical. The enantioselectivity should be observed in comparing the observations on the $R / R$ and the $R / S$ combinations. In many instances, it is only possible to obtain single enantiomers of a chiral compound in pure form. In those instances the control experiments must be performed with the racemic mixture. While the chemistry of the racemic mixture should be identical on both the $R$ - and the $S$-surfaces the enantiospecificity will be revealed by the measurements on the $R / R$ and the $R / S$ adsorbate/surface combinations.

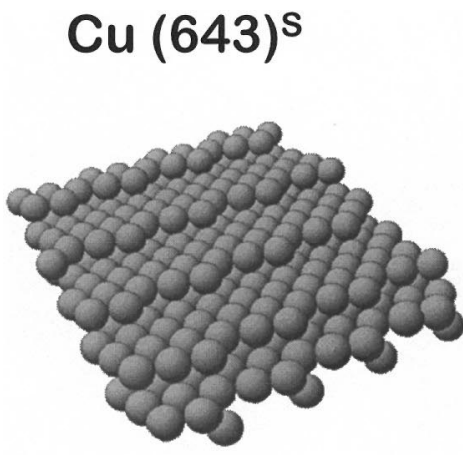

\section{$\mathrm{Cu}(643)^{R}$}

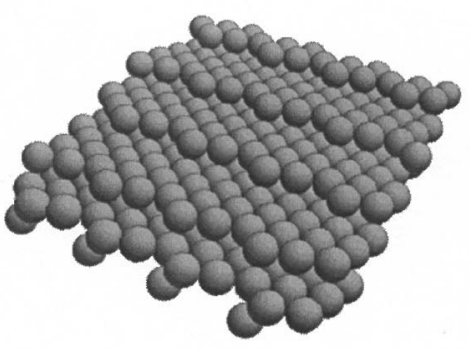

\subsection{R-3-methyl-cyclohexanone desorption from $\mathrm{Cu}(643)^{R}$ and $\mathrm{Cu}(643)^{S}$}

The surfaces used in this work have been the $\mathrm{Cu}(643)^{R}$ and the $\mathrm{Cu}(643)^{S}$. Models of these surfaces and the corresponding LEED patterns are shown in Fig. 1. The surfaces are formed of (111) terraces separated by steps with kinks. The step between kinks is two nearest neighbor spacings in length. It is fairly easy to see that the two surfaces are mirror images of one another and further inspection will reveal that they are not superimposable. Equivalently, one can see that there are no mirror planes in these structures.
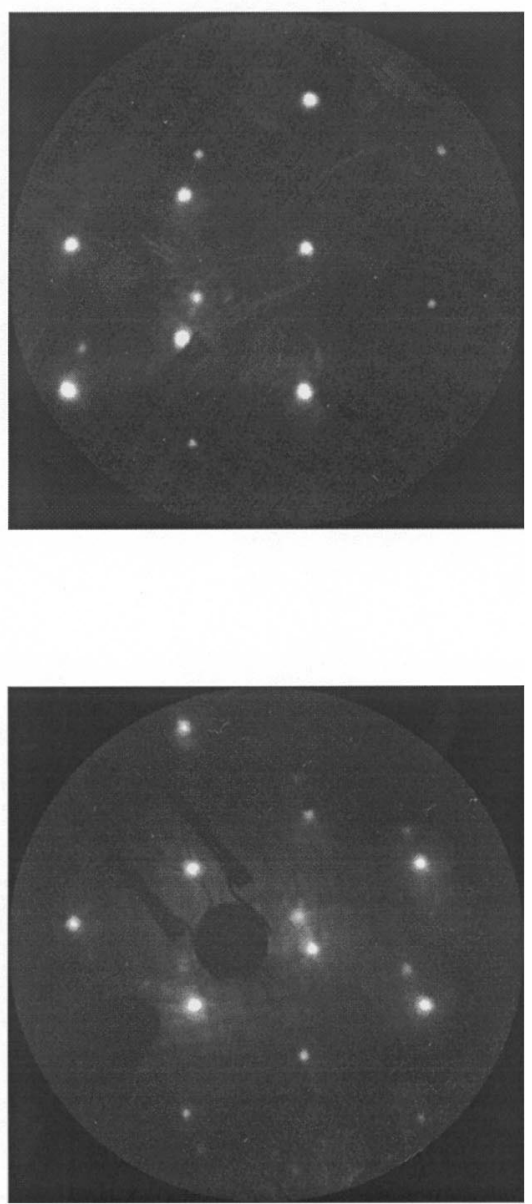

Fig. 1. The LEED patterns obtained from the $\mathrm{Cu}(643)^{R}$ and $\mathrm{Cu}(643)^{S}$ surfaces at an electron beam energy of $183 \mathrm{eV}$. The models for the surface structures are illustrated beside the LEED patterns. The basic features of the LEED patterns are a set of split diffraction spots in a hexagonal array. The splittings of the spots are in directions that are $+23^{\circ}$ and $-23^{\circ}$ from the low index direction. Both the LEED patterns and the surfaces are non-superimposable mirror images of one another. 


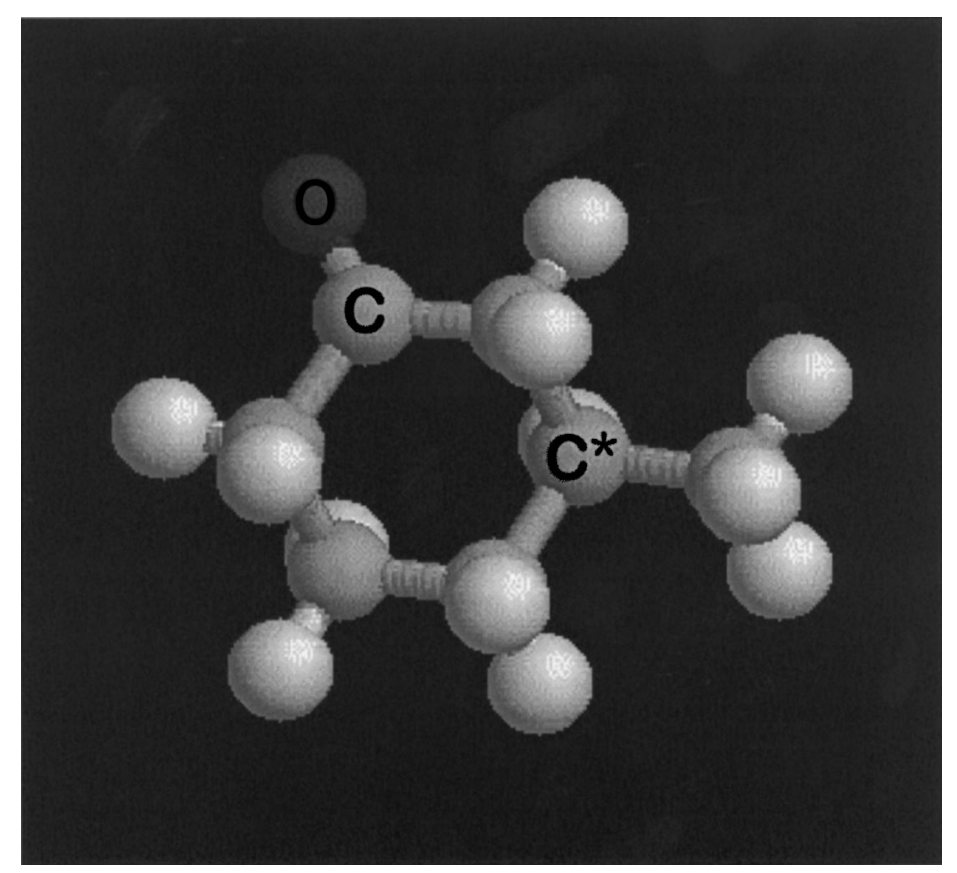

Fig. 2. Ball model of 3-methyl-cyclohexanone. The chiral center is the carbon atom in the $\mathrm{C}_{6}$ ring that is substituted with the methyl group.

The LEED patterns also reveal the handedness of the surfaces. The basic features of the LEED patterns are the pairs of diffraction spots arranged with hexagonal symmetry that results from the (111) orientation of the terraces. The presence of the steps is responsible for the splitting of the spots into pairs and the fact that the steps have kinks, or do not run along low index directions of the crystal, causes the spots to split at angles away from the low index directions in the diffraction pattern. Close inspection of the figure will reveal that these spots are split at $+23^{\circ}$ and at $-23^{\circ}$ from the low index direction. Thus, these LEED patterns are non-superimposable mirror images of one another and directly reflect the handedness of the real space structures.

$R$-3-methyl-cyclohexanone and a racemic mixture of 3-methyl-cyclohexanone have been studied on the $\mathrm{Cu}(643)^{R}$ and the $\mathrm{Cu}(643)^{S}$ surfaces. Fig. 2 shows a model of $R$-3-methyl-cyclohexanone with the chiral center being the carbon atom in the ring attached to the methyl group. The TPD spectra of $R-3-$ methyl-cyclohexanone adsorbed at various coverages on the $\mathrm{Cu}(643)^{R}$ surface are shown in Fig. 3. The basic features of the spectra are two high temperature desorption features at $\sim 345$ and $\sim 380 \mathrm{~K}$ which are present at all coverages and which saturate in intensity as the coverage is increased. These two features are both attributed to desorption from the monolayer adsorbed on the $\mathrm{Cu}(643)^{R}$ surface. At high coverages there is a very low temperature desorption feature at $170 \mathrm{~K}$ which does not appear to saturate with increasing coverage and is believed to be due to multilayer desorption. Initially several ionization fragments were monitored in the mass spectrometer in order to determine whether or not the 3-methyl-cyclohexanone was reacting on the surface. All the fragments exhibited the general shape of the spectra illustrated in Fig. 3 and there was no evidence of decomposition. Furthermore, the relative intensities of the various fragments matched those measured for 3-methyl-cyclohexanone vapor introduced into the chamber background. All indications are that 3-methyl-cyclohexanone adsorbs and desorbs reversibly from the $\mathrm{Cu}(643)^{R}$ and $\mathrm{Cu}(643)^{S}$ surfaces.

The general shape and features of the TPD spectra of $R$-3-methyl-cyclohexanone and a racemic mixture of 3-methyl-cyclochexanone from the $\mathrm{Cu}(643)^{R}$ and the $\mathrm{Cu}(643)^{S}$ surfaces are identical. In order 
TPD - R-3-methyl-cyclohexanone / Cu(643) ${ }^{\mathrm{R}}$ Heating Rate $=1 \mathrm{~K} / \mathrm{sec}$

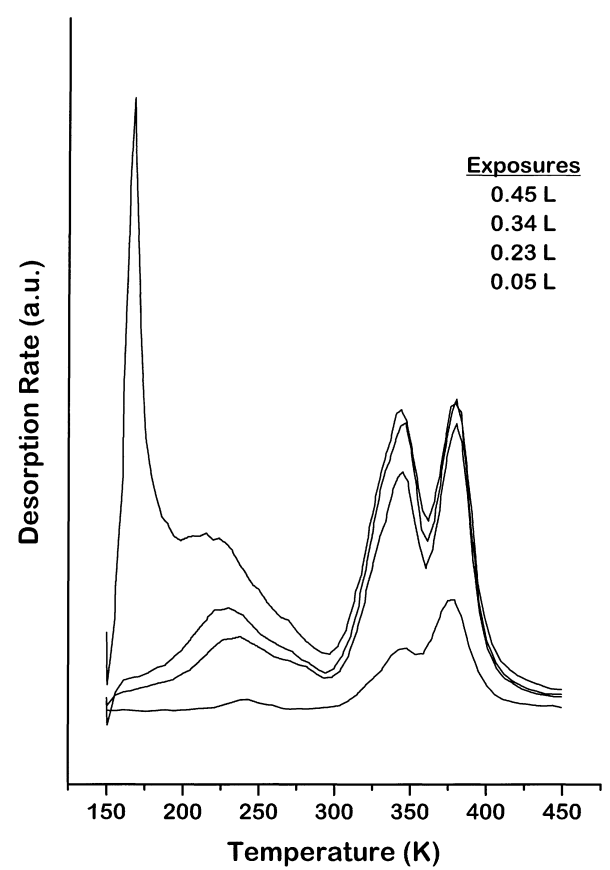

Fig. 3. Thermally programmed desorption spectra of 3-methylcyclohexanone on the $\mathrm{Cu}(643)^{R}$ surface. The two high temperature desorption peaks are attributed to desorption of molecules bonded directly to the surface. The multilayer desorption feature is at $170 \mathrm{~K}$.

to identify enantiospecificity in the desorption kinetics we have carefully measured the peak desorption temperatures for the high temperature desorption peak obtained and for all four combinations of racemic 3-methyl-cyclohexanone and $R$-3-methyl-cyclohexanone on the $\mathrm{Cu}(643)^{R}$ and the $\mathrm{Cu}(643)^{S}$ surfaces. The high temperature peak corresponds to the lower coverage on the surface and thus, the adsorbed molecules are most likely to be interacting with the kinked steps. In order to do this most accurately we have taken the desorption data lying within $\pm 10 \mathrm{~K}$ of each peak maximum and then used a Gaussian function to fit the shape of the peak and yield the peak position. This approach has enabled us to estimate the desorption peak temperatures with a standard deviation of roughly $0.5 \mathrm{~K}$. For each adsorbate-substrate combination the desorption experiment has been
Table 1

The peak desorption temperature measured using the racemic mixture of $R$ - and $S$-3-methyl-cyclohexanone (Rac) and the enantiomerically pure $R$-3-methyl-cyclohexanone $(R)$ on the $\mathrm{Cu}(643)^{R}$ and $\mathrm{Cu}(643)^{S}$ surfaces

\begin{tabular}{ll}
\hline Molecule/surface & Peak desorption temperature (K) \\
\hline$R a c / C u(643)^{R}$ & $385.7 \pm 0.6$ \\
$R a c / C u(643)^{S}$ & $385.3 \pm 0.4$ \\
$R / C u(643)^{R}$ & $383.1 \pm 0.4$ \\
$R / C u(643)^{S}$ & $386.4 \pm 0.7$ \\
\hline
\end{tabular}

repeated six or seven times using a coverage of the 3-methyl-cyclohexanone that saturates both the high temperature desorption peaks but does not produce the multilayer. The surfaces were cleaned between experiments. This has yielded six or seven points with which to calculate an average and a standard deviation for the desorption peak temperatures (Table 1). These have then been converted into estimates of the desorption energy $\left(\Delta E_{\mathrm{des}}\right)$ by using Redhead's equation and an assumption that the pre-exponential factor is $v=$ $10^{13} \mathrm{~s}^{-1}$ [9]. These desorption energies are plotted in Fig. 4. It is important to note that while there may be systematic error in the absolute magnitudes of these desorption energies due to the assumptions that go into the Redhead analysis, their relative magnitudes and the fact that there are significant differences are what is important to this study. The desorption energies for the racemic mixtures from both the $\mathrm{Cu}(643)^{R}$ and the $\mathrm{Cu}(643)^{S}$ surfaces are identical, as they should be. The important feature of these data is that the desorption energies for $R$-3-methyl-cyclohexanone on the $\mathrm{Cu}(643)^{R}$ and the $\mathrm{Cu}(643)^{S}$ surfaces differ by $\Delta \Delta E_{\text {des }}=0.22 \pm 0.05 \mathrm{kcal} / \mathrm{mole}$ and that this difference is significant. The desorption spectra of $R$-3-methyl-cyclohexanone reveal for the first time the enantiospecific differences in the desorption kinetics of a chiral molecule on the $R$ - and $S$-enantiomers of a chiral single crystal surface.

\section{2. $R$ - and S-2-butanoxy orientation on $A g(643)^{R}$}

In previous work we studied the surface chemistry of chiral 2-butanoxy groups on the chiral $\operatorname{Ag}(643)^{R}$ and $\operatorname{Ag}(643)^{S}$ surfaces [4]. As with alkoxy groups on many metal surfaces these decompose during heating by $\beta$-hydride elimination to yield methylethylketone 


\section{Desorption Energies for Racemic and R-3-methylcyclohexanone on $\mathrm{Cu}(643)^{\mathrm{R}}$ and $\mathrm{Cu}(643)^{\mathrm{S}}$}

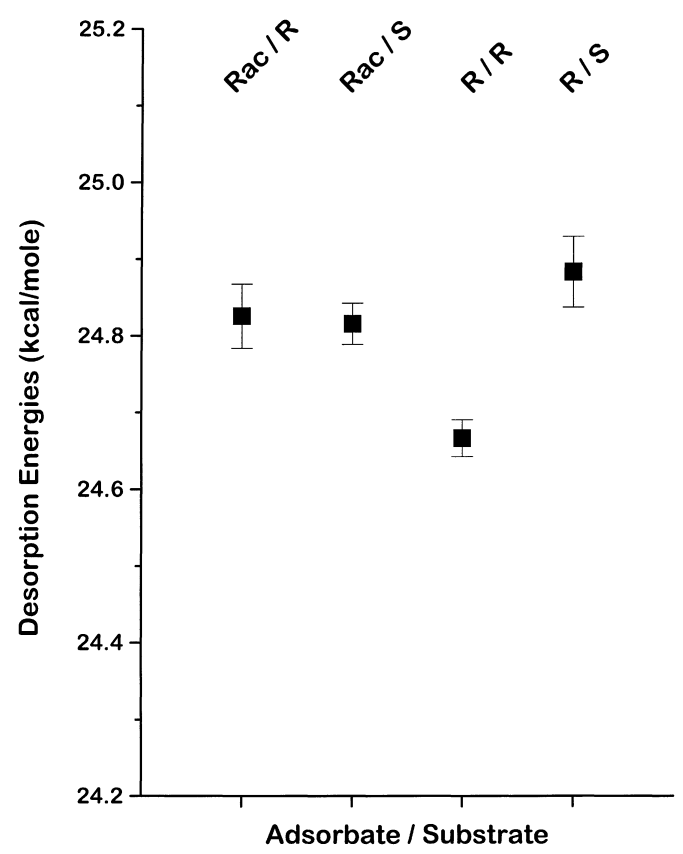

Fig. 4. Plot of the desorption energies of $R$-3-methyl-cyclohexanone and a racemic mixture of $R$ - and $S$-3-methyl-cyclohexanone adsorbed on the $\mathrm{Cu}(643)^{R}$ and the $\mathrm{Cu}(643)^{S}$ surfaces. The desorption energies of the racemic mixture are identical on the two surfaces for both desorption peaks. The desorption energies of the $R$-3-methyl-cyclohexanone differ significantly on the two surfaces clearly revealing enantiospecificity.

which desorbs rapidly from the surface. In that work it was not possible to distinguish between the kinetics of $\beta$-hydride elimination for $R$ - and $S$-2-butanoxy decomposition on either of the $\operatorname{Ag}(643)^{R}$ or $\operatorname{Ag}(643)^{S}$ surfaces. The implications were that the enantiospecific differences in the activation barriers for $\beta$-hydride elimination among these four adsorbate-substrate combinations were less than $0.1 \mathrm{kcal} / \mathrm{mole}$.

In addition to reaction and desorption kinetics that ought to exhibit enantioselectivity for chiral molecules on chiral surfaces there are other properties that ought to be enantiospecific. One such property is molecular orientation of the adsorbed species. The orientation of a chiral molecule with respect to the surface must be dependent on the handedness of the local environment in which it sits.

The most sensitive probe of molecular orientation of adsorbates on metal surfaces is probably infrared absorption. The intensity of the absorption of a vibrational mode for a molecule on a surface is dependent on the angle $\left(\theta_{\vec{\mu} \hat{n}}\right)$ between the dynamic dipole moment $(\vec{\mu})$ associated with that mode and the surface normal $(\hat{n})[8,10]$. The absorption intensity has the functional form

$I \propto(\vec{\mu} \hat{n}) \propto \cos ^{2}\left(\theta_{\vec{\mu} \hat{n}}\right)$.

As a result modes with their dynamic dipoles oriented parallel to the surface do not absorb while those that have their dipoles oriented along the surface normal are most intense. Usually it is not easy to reproduce the exact absorption intensities from experiment to experiment, however, the relative intensities of absorption features within a spectrum are more easily reproduced and are indicative of orientation.

The FT-IRRAS spectra of $R$ - and $S$-2-butanoxy groups on the $\operatorname{Ag}(643)^{R}$ surface are shown in Fig. 5. These are complicated spectra and no attempt has been made to fully assign all the modes observed. That is not the point of this work. Instead we are most interested in the relative intensities of the modes. First note the level of reproducibility of some of the relative intensities. In the $\mathrm{C}-\mathrm{H}$ stretch regions from 2900 to $3000 \mathrm{~cm}^{-1}$ the relative intensities of the major bands are reproduced exactly. Even some of the fine structure on these peaks it reproduced. In the lower frequency region of the $\mathrm{C}-\mathrm{C}$ stretches and the deformations $\left(900-1100 \mathrm{~cm}^{-1}\right)$ the relative intensities of the modes are again reproduced quite well. There are however, two sets of bands for which the relative intensities of the absorption spectra are inverted between the two spectra. The first pair are at 2855 and $2875 \mathrm{~cm}^{-1}$ and arise from C-H stretches. The second pair at 1378 and $1475 \mathrm{~cm}^{-1}$ correspond to the $\mathrm{CH}_{2}$ scissors mode and the $\mathrm{CH}_{3}$ symmetric deformation. The fact that the relative intensities of these modes differ between the two enantiomers is indicative of the fact that the projections of their dynamic dipoles onto the surface normal differ. This is clear evidence that the orientations of $R$ - and $S$-2-butanoxy groups on the $\operatorname{Ag}(643)^{R}$ are different. This again is a demonstration of enantiospecific chemistry on these chiral surfaces. 


\section{FT-IRAS on $A g(643)^{R}$}

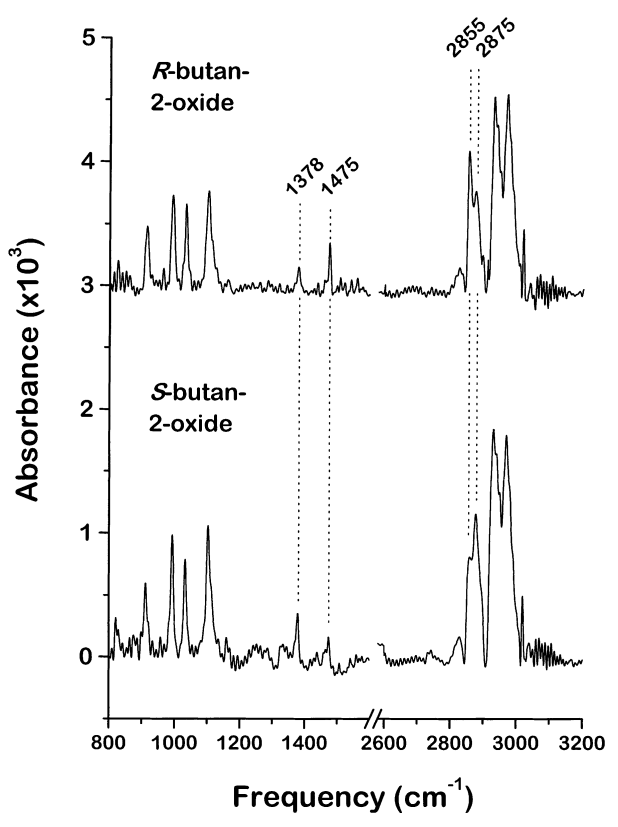

Fig. 5. The FT infrared absorption spectra of $R$ - and $S$-2-butanoxy groups on the $\operatorname{Ag}(643)^{R}$ surface. A number of the absorption bands in the regions around $2900-3000 \mathrm{~cm}^{-1}$ and in the region $900-1100 \mathrm{~cm}^{-1}$ exhibit highly reproducible relative intensities. The pairs of bands at 1378 and $1475 \mathrm{~cm}^{-1}$ and at 2855 and $2875 \mathrm{~cm}^{-1}$ have relative intensities that invert between the two enantiomers of the adsorbate. These differences in the intensities reveal that the adsorption geometries of the two enantiomers differ and therefore, must be sensitive to the chirality of the surface.

\section{Discussion}

There are now several demonstrations of the enantiospecific properties of chiral high Miller index single crystal metal surfaces. The LEED patterns of the $\mathrm{Cu}(643)$ surfaces shown here and of other chiral surfaces indicate that they can indeed be prepared such that they have long range order with chiral structures. The other attempts to observe enantiospecific properties of chiral surfaces have made use of $\mathrm{Ag}$ and of Pt surfaces and have also used LEED to demonstrate the order and orientation of the surfaces. Of course, it may not always be the case that such high Miller index expected on the basis of bulk termination. Our work has probably benefited from the foresight of using surfaces that are vicinal to the most stable (111) planes of the fcc lattice. Other such high Miller index surfaces can undoubtedly reconstruct although it is not clear that reconstruction necessarily removes chirality.

The evidence for enantiospecific properties of chiral surfaces now includes measured electrooxidation rates for D- and L-glucose [4,7], observations of enantiospecific adsorbate orientations, and both experimental and theoretical observations $[5,6]$ of enantioselective desorption energies. One of the issues that is of interest is the general nature of molecules which will exhibit enantiospecific properties on such surfaces. There have been two failed attempts to observe enantiospecific desorption kinetics using $R$ - and $S$-2-butanol [3] and 1-chloro-2-methyl-butane [11], before the current study using $R$-3-methyl-cyclohexanone. With this limited data set it can only appear that observing enantiospecific surface chemistry will require molecular complexity that is much greater than that usually used in surface science experiments. This is perhaps not to surprising and is certainly supported by the experiments with D- and L-glucose which observed enantioselective electrooxidation kinetics.

Finally, it is probably worth discussing the magnitudes of enantiospecificity in desorption energies or reaction barriers that become interesting from the point of view of controlling enantioselectivity. The difference in desorption energies for $R$-3-methylcyclohexanone from the $\mathrm{Cu}(643)^{R}$ and the $\mathrm{Cu}(643)^{S}$ surfaces is $\Delta \Delta E_{\mathrm{des}}=0.22 \pm 0.05 \mathrm{kcal} / \mathrm{mole}$. This translates into a difference in desorption rates of about $30 \%$ at room temperature. An enantiospecific differences of $2 \mathrm{kcal} / \mathrm{mole}$ would correspond to a factor of 30 difference in desorption rates. Thus while huge difference in desorption energies cannot be expected in experiments of the type described here, they are not necessary from the perspective of enantioselectivity.

\section{Acknowledgements}

This work was supported by NSF Grant CTS9813937.

\section{References}

[1] G. Jannes, V. Dubois (Eds.), Chiral Reactions in Heterogeneous Catalysis, Plenum Press, New York, 1995. 
[2] S.C. Stinson, Chiral drug interactions, Chem. Eng. News (1999) 101.

[3] C.F. McFadden, P.S. Cremer, A.J. Gellman, Langmuir 12 (1996) 2483.

[4] A. Ahmadi, G. Attard, J. Feliu, A. Rodes, Langmuir 15 (1999) 2420.

[5] D.S. Sholl, Langmuir 14 (1998) 862-867.

[6] T.D. Power, D.S. Sholl, J. Vacuum Sci. Technol. A 17 (1999) 1700.
[7] G.A. Attard, A. Ahmadi, J. Feliu, A. Rodes, E. Herrero, S. Blais, G. Jerkiewicz, J. Phys. Chem. B 103 (1999) 1381.

[8] S.C. Street, A.J. Gellman, Quantitative adsorbate orientation from vibrational spectra: ethoxides on $\mathrm{Cu}\left(\begin{array}{lll}1 & 1 & 1\end{array}\right)$, J. Chem. Phys. 105 (1996) 7158.

[9] P.A. Redhead, Vacuum 12 (1962) 203.

[10] Y.J. Chabal, Surf. Sci. Rep. 8 (1988) 211.

[11] J.D. Horvath, A.J. Gellman, submitted for publication. 